\title{
Ethanol extract of Lycoris radiata induces cell death in B16F10 melanoma via p38-mediated AP-1 activation
}

\author{
MINSIK SON ${ }^{1 *}$, AEYUNG KIM $^{2 *}$, JAEWOO LEE$^{1}$, CHUL-HONG PARK $^{1}$, \\ JIN-CHUL HEO ${ }^{3}$, HYUN-JIN LEE ${ }^{1}$ and SANG-HAN LEE ${ }^{1,3}$ \\ ${ }^{1}$ Department of Food Science and Biotechnology, Kyungpook National University, Daegu 702-701;
${ }^{2}$ Department of Molecular Science and Technology, Ajou University, Suwon 443-749;
${ }^{3}$ Food and Bio-Industry Research Institute, Kyungpook National University, Daegu 702-701, Korea
}

Received October 7, 2009; Accepted January 18, 2010

DOI: $10.3892 /$ or_00000881

\begin{abstract}
Some active alkaloids isolated from Lycoris, a bulbous perennial herb, was shown to possess various antitumor and anti-inflammatory activities. In this study, we evaluated the in vitro apoptotic effect of ethanol extract from Lycoris radiata (LRE) and further probed the underlying molecular mechanisms of LRE effects. The survival rate of B16F10 melanoma cells exposed to LRE was decreased in a dose-dependent manner, cell growth was retarded by arresting cell cycle at $\mathrm{G}_{1}$ phase and apoptotic appearance such as caspase-3 activation as well as DNA fragmentation was observed by LRE treatment. In addition, LRE induced p38 and c-Jun phosphorylation, followed by activation of transcription factor AP-1. Pretreatment with the p38 inhibitor (SB203580) blocked LRE-induced AP-1 transcriptional activity, and curcumin, AP-1 inhibitor, dramatically inhibited LRE-induced apoptosis in B16F10 melanoma cells. Our results collectively indicate that LRE-mediated apoptosis occurs through the activation of p38 and AP-1 pathway and potentially LRE exhibits anti-cancer activity against B16F10 melanoma cells.
\end{abstract}

\section{Introduction}

The better understanding of the means of overcoming unlimited proliferation or inducing apoptosis against a variety of malignancies raises new hope of improving prognosis for patients with cancer (1-3). Apoptosis is an essential phenomenon for many physiological events including maintenance of normal tissue and aging $(4,5)$. In

Correspondence to: Professor Sang-Han Lee, Department of Food Science and Biotechnology, Kyungpook National University, Daegu 702-701, Korea

E-mail: sang@knu.ac.kr

*Contributed equally

Key words: Lycoris radiata, melanoma, apoptosis, cell cycle arrest, AP-1, p38 addition, apoptosis is also linked with pathological conditions such as inflammation and tumorigenesis $(4,6,7)$. Therefore, identification of the regulating mechanisms for apoptosis may be important for therapeutic considerations.

The genus Lycoris is a small group of Amaryllidaceae composed of 20 species and distributed in the moist warm temperate woodlands of eastern Asia including Korea (8). Some alkaloids extracted from amaryllis plants have been shown to exhibit various natural anti-tumor activities. Lycorine isolated from Amaryllidaceae (Lycoris) has been demonstrated to suppress cell growth of the human leukemia cell line HL-60 (9) as well as the multiple myeloma cell line KM3 (10) by arresting the cell cycle, subsequently inducing apoptosis of tumor cells. It has been found that lycorine-mediated apoptosis was triggered through the cytochrome c-mediated and caspase-dependent pathway (9-11). Additionally, reduction of bcl-2 family proteins was found. Another recent study reported that lycorine causes a rapid turnover of protein levels of myeloid cell leukemia-1 (Mcl-1), which may play an important survival role in a variety of tumor cells including leukemia (11). These results collectively suggest that lycorine may be a good candidate therapeutic agent against leukemia. Besides lycorine, the search for bioactive constituents from the bulbs of Lycoris radiata such as lycoranines A and B is in progress (12). Though apoptosisinducing effect of Lycoris is well known, the specific molecular mechanisms governing Lycoris-induced apoptosis are poorly understood.

In the present study, we examined the effect of ethanol extract from Lycoris radiata (LRE) as anti-cancer potential using B16F10 murine melanoma cell line and explored its possible molecular mechanisms. Our present studies demonstrate that LRE is potent in inhibiting the growth by $\mathrm{G}_{1}$ cell cycle arrest and inducing apoptosis in B16F10 melanoma cells. In addition, we found that p38-mediated AP1 activation plays an essential role in LRE-mediated apoptosis.

\section{Materials and methods}

Cell lines and culture. The murine B16F10 melanoma cell line was grown in Dulbecco's modified Eagle's medium (DMEM; Gibco/Invitrogen, Grand Island, NY) supplemented with $10 \%$ 
heat-inactivated fetal bovine serum (FBS; Gibco/Invitrogen), $0.01 \%$ penicillin/streptomycin (Gibco/Invitrogen), at $37^{\circ} \mathrm{C}$ in a humidified $5 \% \mathrm{CO}_{2}$ incubator. The immortal murine melanocyte cell line from C57BL/6 mouse, Melan-a, was kindly provided by Dr Dorothy Bennett (St. George's Hospital Medical School, London, UK). Melan-a cells were maintained in RPMI-1640 supplemented with $10 \%$ FBS, 2 mM L-glutamine, $50 \mathrm{mg} / \mathrm{l}$ gentamicin, $0.1 \mathrm{mM}$ 2-mercaptoethanol and $200 \mathrm{nM}$ phorbol 12-myristate 13-acetate with $10 \% \mathrm{CO}_{2}$.

Antibodies and chemicals. Goat polyclonal anti-Akt, anti-actin and rabbit polyclonal anti-p38 were purchased from Santa Cruz Biotechnology (Santa Cruz, CA). Rabbit polyclonal anti-phospho-Akt, anti-phopho-c-jun and anti-c-jun were obtained from Cell Signaling Technology (Beverly, MA) and anti-phospho-p38 was purchased from ABR (Affinity BioReagents, Golden, CO). We obtained mouse monoclonal anti-caspase-3 and anti-PARP from Alexis (San Diego, CA). Secondary horseradish peroxidase (HRP)-conjugated antirabbit, anti-goat, and anti-mouse antibodies were acquired from Santa Cruz Biotechnology. SB203580 and curcumin were obtained from Calbiochem (La Jolla, CA) and Sigma Chemical Co. (St. Louis, MO), respectively.

Preparation of ethanol extract from Lycoris radiata. The ethanol extract of LR (LRE) was obtained from the bulb of this plant collected in South Korea. Air-dried powder from LR bulbs was sequentially extracted by water and $50 \%$ ethanol at $60^{\circ} \mathrm{C}$ for $24 \mathrm{~h}$. The aqueous ethanol extract was obtained by centrifugation at $18,000 \mathrm{x} \mathrm{g}$ for $30 \mathrm{~min}$ at $4^{\circ} \mathrm{C}$ and then concentrated to dryness in a lyophilizer. The dried preparation was dissolved in DMSO at concentration of $100 \mathrm{mg} / \mathrm{ml}$ (stock solution) and filtered through a $0.20 \mu \mathrm{m}$ disk filter (Sartorius Stedim Biotech, Germany).

Cell viability assay. The effect of LRE extract on cell viability and cellular metabolic activity were examined using trypan blue dye exclusion and 3-[4,5-dimethylthiazol-2-yl]-2,5diphenyl-tetrazolium bromide (MTT; Amresco, Solon, OH) assay, respectively. In MTT assay, formazan precipitates were dissolved in dimethyl sulfoxide (DMSO), and the absorbance were measured with Victor $3^{\mathrm{TM}} 1420$ Multilabel Counter (Perkin-Elmer, Waltham, MA).

Flow cytometry detection of cell cycle and apoptosis. To detect the distribution of cell cycle, LRE-treated cells were harvested, washed twice with PBS and fixed with ice-cold $70 \%$ ethanol at $4^{\circ} \mathrm{C}$ for $24 \mathrm{~h}$. After washing with PBS, intracellular DNA was labeled with $1 \mathrm{ml}$ of cold propidium iodide (PI) solution (0.1\% Triton X-100, $0.1 \mathrm{mM}$ EDTA, $0.05 \mathrm{mg} / \mathrm{ml}$ RNase A, $50 \mu \mathrm{g} / \mathrm{ml}$ PI in PBS) on ice for $30 \mathrm{~min}$ in the dark. Apoptotic cells were determined using Annexin V-FITC apoptosis detection kit (BD Biosciences, San Diego, CA). After LRE treatment, cells were washed twice in ice cold phosphatebuffered saline (PBS) and suspended in $500 \mu 1$ of binding buffer and incubated with $1 \mu \mathrm{l}$ of Annexin V-FITC in the dark for $15 \mathrm{~min}$ at room temperature. Cell cycle distribution and Annexin V-positive apoptotic cells were measured with FACSCalibur flow cytometry (Beckton-Dickinson, NJ) and analyzed by Cell Quest software.
TdT-mediated dUTP nick end-labeling (TUNEL) assay. DeadEnd $^{\mathrm{TM}}$ Fluorometric TUNEL System (Promega, Madison, WI) was used to measure nuclear DNA fragmentation in apoptotic cells. Briefly, cells $\left(1 \times 10^{4}\right.$ cells $\left./ 0.2 \mathrm{ml} \mathrm{PBS}\right)$ attached to microscope slide by cytospin were fixed with $4 \%$ methanolfree formaldehyde in PBS for $25 \mathrm{~min}$ at $4^{\circ} \mathrm{C}$, washed twice with PBS and treated with permeabilization solution $(0.2 \%$ Triton X-100 in PBS) for 5 min. After equilibration, cells were labeled with $\mathrm{TdT}$ reaction mix for $60 \mathrm{~min}$ at $37^{\circ} \mathrm{C}$ in the dark and then reaction was stopped by addition of $2 \mathrm{X} \mathrm{SSC}$. Cells were counterstained with Vectashield (mounting medium with DAPI, Vector Laboratories, Burlingame, CA) and localized green fluorescence of apoptotic cells was detected by fluorescence microscope.

Western blot analysis. Whole cell lysates were prepared using M-PER Mammalian Protein Extraction Reagent (Pierce Biotechnology, Rockford, IL) and the protein concentration was determined using the BCA assay. Proteins were visualized after immunoblotting using a PowerOpti-ECL Western blotting Detection reagent (Animal Genetics, Inc. Korea) and a LAS 3000 imaging system (Fujifilm Corporation, Tokyo, Japan).

Luciferase reporter assay. Semi-confluent cells grown in 12-well plates were co-transfected with AP-1 luciferase reporter plasmid and $\mathrm{pCMV- \beta -galactosidase} \mathrm{reporter} \mathrm{plasmid}$ using Lipofectamine 2000 (Invitrogen). After incubation, cells were harvested in passive lysis buffer and the luciferase activities were measured on a Victor3 1420 multilabel counter with the luminescence microplate reader set (Perkin Elmer, Victor ${ }^{\mathrm{TM}}$ ) using the Luciferase Assay System according to the manufacturer's instructions (Promega). The $ß$-galactosidase activity was measured using $o$-nitrophenyl B-galactopyranoside as a substrate and used to control the variability in transfection efficiency. Luciferase activity was normalized with the $\beta$-galactosidase activity.

Electrophoretic mobility shift assay (EMSA). Nuclear extracts were prepared after treatment with LRE for the indicated times and protein-DNA binding activity was determined using Panomics' AP-1 EMSA kit (EMSA Gel Shift Kit, Fremont, $\mathrm{CA}$ ) according to the manufacturer's instructions.

Statistical analysis. Results are presented as means \pm standard deviation (SD). A standard two-tailed t-test was used for statistical analysis. P-values of $<0.05$ were considered significant.

\section{Results and Discussion}

LRE treatment reduces cell growth in dose-dependent manner and induces $G_{I}$ cell cycle arrest in B16F10 melanoma cells. To investigate the effect of LRE treatment on tumor cell growth, $60-70 \%$ confluent B16F10 cells were incubated with various concentrations of LRE for $24 \mathrm{~h}$. As shown in Fig. 1A, LRE treatment reduced cell density in comparison to untreated B16F10 cells and elicited moderate apoptotic appearance. The survival rate of $\mathrm{B} 16 \mathrm{~F} 10$ cells exposed to the increasing concentration of LRE (10-50 $\mu \mathrm{g} / \mathrm{ml})$ for $24 \mathrm{~h}$ was reduced in a dose-dependent manner and anti-proliferative activity of 
A

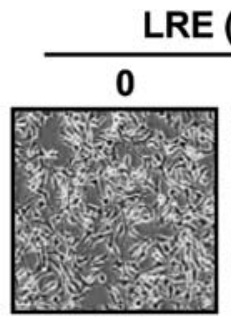

25
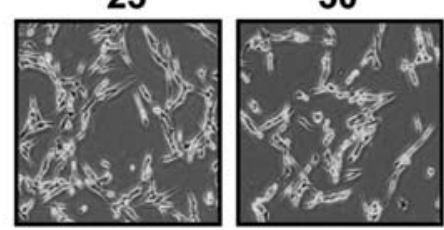

B

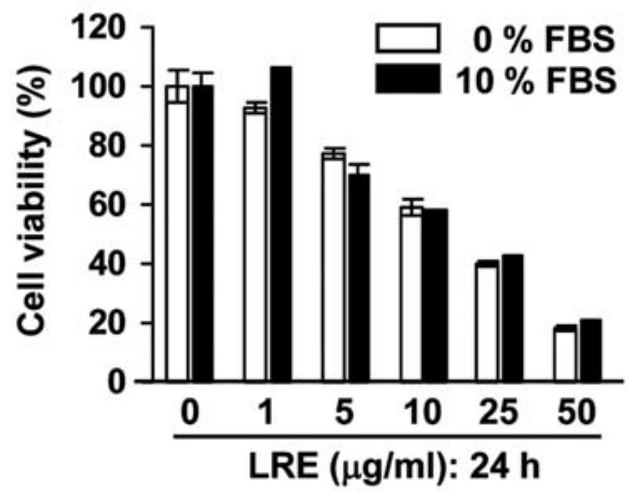

C

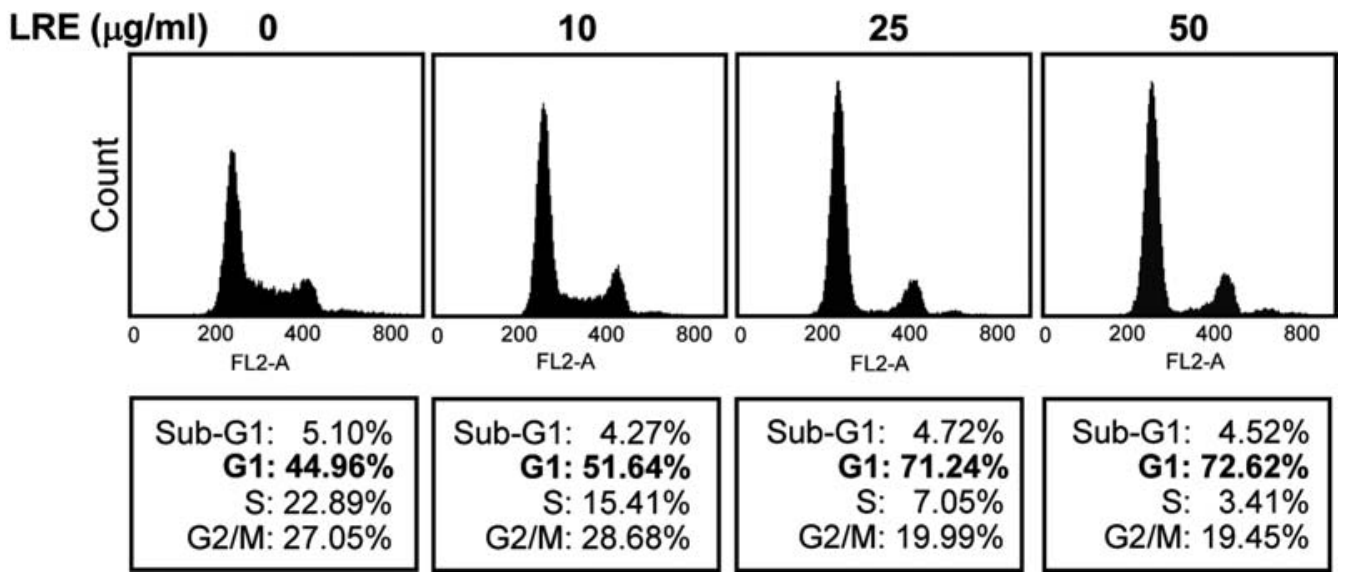

Figure 1. Reduction of cell viability and induction of cell cycle arrest at $\mathrm{G}_{1}$ phase in B16F10 melanoma cells by LRE treatment. (A) B16F10 cells were incubated with indicated concentrations of LRE for $24 \mathrm{~h}$ and then cell morphology was observed using a phase contrast microscope. (B) Cell viability after LRE treatment in the presence or absence of FBS was determined using the MTT assay and represented as percentage value relative to untreated cells. Data are expressed as the mean \pm SD. (C) Cells treated with LRE for $12 \mathrm{~h}$ were stained with propidium iodide and determined cell cycle distribution by flow cytometry analysis.

LRE was observed in both conditions with or without serum (Fig. 1B). To further investigate the underlying basis of the anti-proliferative activity of LRE, cell cycle distribution was examined. Treatment of B16F10 cells with LRE for $12 \mathrm{~h}$ resulted in the increase of $G_{1}$ phase compared to untreated control cells and this increase was accompanied by a corresponding reduction in the percentage of cells in $\mathrm{S}$ phase (Fig. 1C). The percentage of $\mathrm{G}_{1}$ phase in LRE $(25 \mu \mathrm{g} / \mathrm{ml})$ treated cells was $71.24 \%$, whereas $44.96 \%$ in untreated control cells while S phase in LRE-treated cells was $7.05 \%$, whereas $22.89 \%$ in control cells. These data suggest that inhibition of cell proliferation in B16F10 cells by LRE may be exerted by the induction of $G_{1}$ phase arrest.

LRE treatment induces apoptosis in B16F10 melanoma cells via caspase-3 activation. As a decrease in cell proliferation may result from the induction of apoptosis, we first investigated whether LRE treatment increases the apoptotic marker Annexin V using flow cytometry. As shown in Fig. 2A, untreated control cells contained negligible $(0.99 \%)$ apoptotic cells, while LRE-treated cells showed remarkable increase in the Annexin V-positive population in a dose-dependent manner. To confirm the effect of LRE on the apoptosis, nuclear DNA fragmentation through the action of endogenous endonuclease was measured by fluorometric TUNEL system. Under fluorescence microscope, considerable amount of fluorescein-12-dUTP-labeled DNA was noted only in cells treated with $25 \mu \mathrm{g} / \mathrm{ml}$ of LRE (Fig. 2B). These green fluorescent apoptotic cells were localized in the DAPIpositive nucleus. We also examined the Akt expression and its phosphorylation in $\mathrm{B} 16 \mathrm{~F} 10$ cells after LRE treatment for $24 \mathrm{~h}$. As shown in Fig. 3A, LRE decreased the phosphorylation of Akt in a dose-dependent manner, with substantial inhibition already evident at the dose of $10 \mu \mathrm{g} / \mathrm{ml}$. Moreover, cleaved forms of caspase- 3 and its substrate PARP were also detected in LRE-treated cells. These data suggest that reduction of cell viability in B16F10 cells by LRE may be exerted by the induction of $\mathrm{G}_{1}$ phase cell cycle arrest and subsequent apoptotic process accompanying caspase- 3 activation.

LRE-induced apoptosis involves the activation of p38-MAPK and AP-1 in B16F10 cells. To explore the signaling pathway 
A

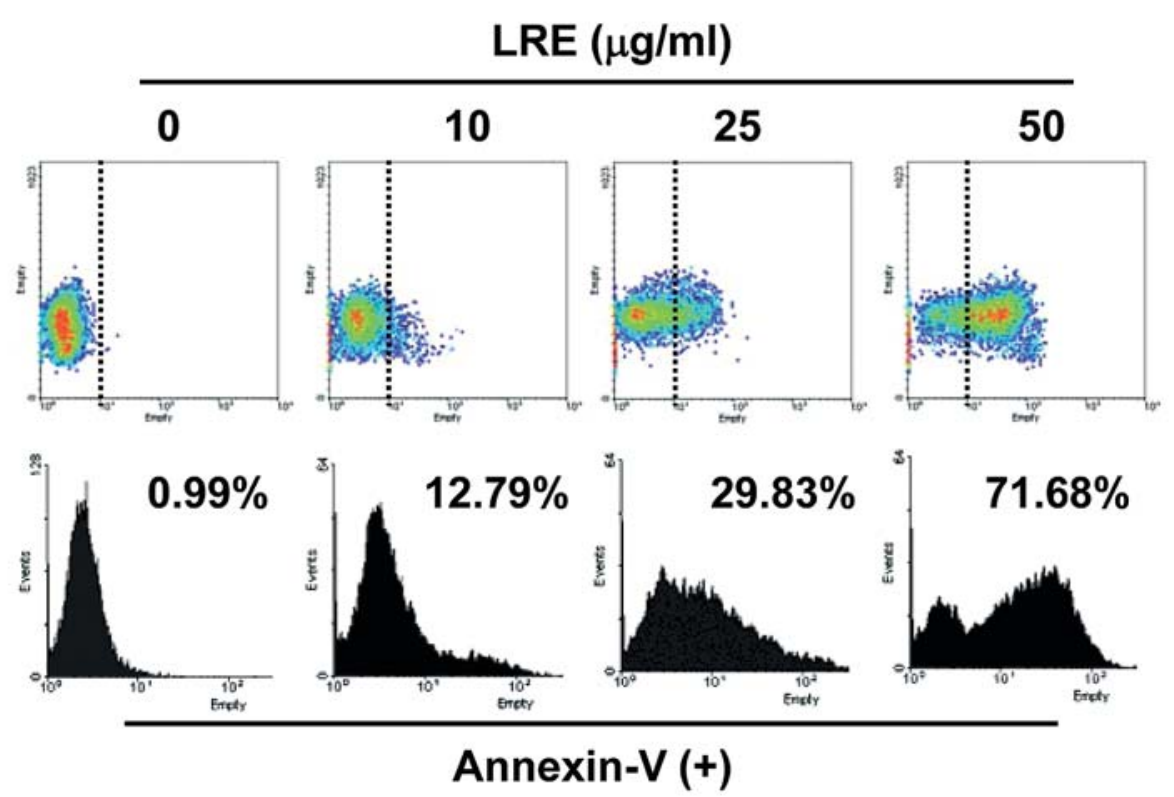

B

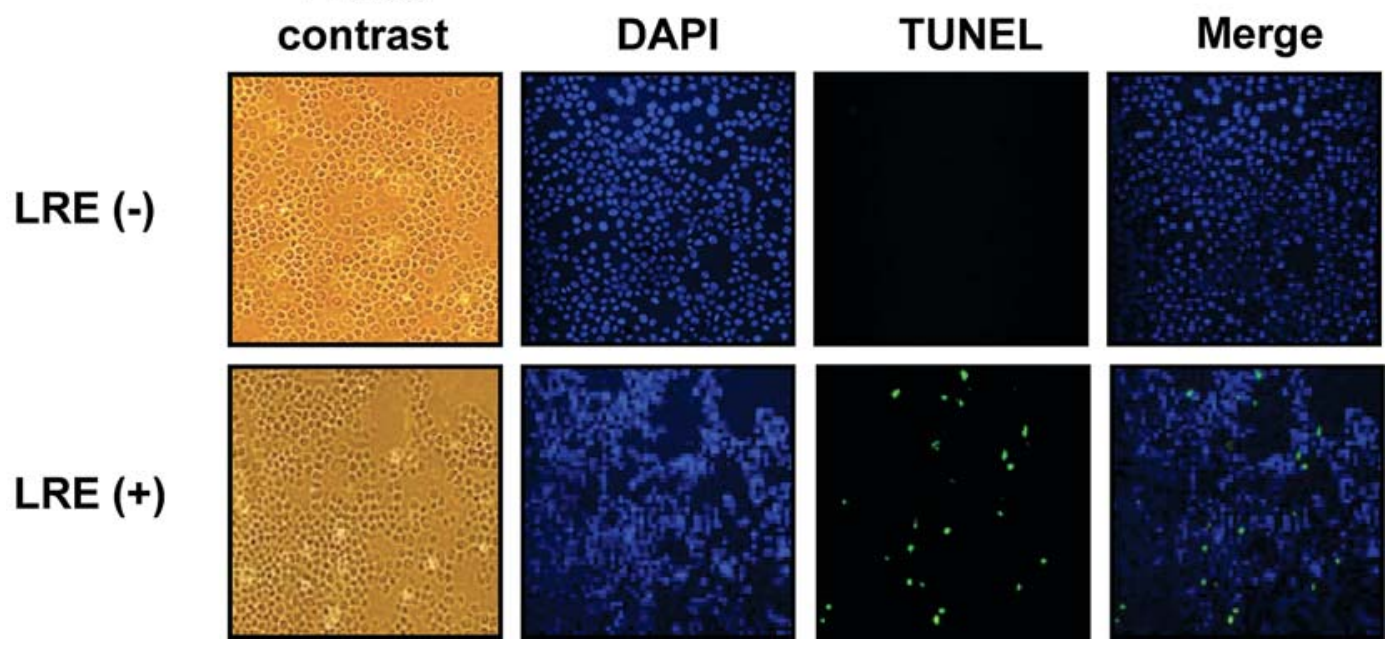

Figure 2. Apoptosis induction by LRE treatment. (A) B16F10 cells were treated with indicated concentrations of LRE for 24 h, stained with Annexin V and analyzed by flow cytometry. Results are from a single analysis, representative of two independent experiments. (B) Cells exposed to $25 \mu \mathrm{g} / \mathrm{ml}$ of LRE for $24 \mathrm{~h}$ were smeared on the slide glass by cytospin and apoptotic DNA end was detected by TUNEL assay. Stained cells were mounted with a mounting medium containing DAPI and observed using a fluorescence microscope (original magnification, x100). Cell images are representative of two independent experiments.

involved in LRE-mediated apoptosis, the changes in the phosphorylation of p38 and ERK were examined after LRE treatment for the indicated time. Phosphorylation of $\mathrm{p} 38$ by LRE was distinct after $30 \mathrm{~min}$ and declined to control level immediately (Fig. 3B). Significant phosphorylation of ERK could not be observed under similar experimental conditions (data not shown). MAPK signaling pathways have been shown to affect AP-1 activity which is an important regulatory protein involved in cell growth, differentiation, transformation and apoptosis $(13,14)$. AP-1 is composed as a homo- or hetero-dimeric complex of c-Jun, JunB, JunD and c-Fos transcription factors and their activities are controlled by transcriptional regulation or by phosphorylation $(15,16)$. As shown in Fig. 3B, the phosphorylation of c-Jun at Ser63 was induced by LRE treatment. In addition, LRE increased AP-1 promoter activity in a concentration-dependent manner by $\sim 10$-fold at a dose of $50 \mu \mathrm{g} / \mathrm{ml}$ as assessed by luciferase reporter gene assay (Fig. 3C) and this increase of AP-1 activity seemed to be caused by the modulation of c-Jun phosphorylation. The DNA-binding activity of AP-1 detected by EMSA was also significantly increased by LRE treatment for $3 \mathrm{~h}$ compared to untreated control cells (Fig. 3D).

Pretreatment with p38 inhibitor completely blocks LREmediated AP-1 activation and AP-1 inhibitor retains cell viability even after LRE treatment. To characterize AP-1 activation pathway in response to LRE treatment, the effect of pretreatment of p38 inhibitor (SB203580) prior to incubation with LRE on the AP-1 activity was examined. As shown in Fig. 4A, LRE-mediated increase in AP-1 promoter activity was 
A

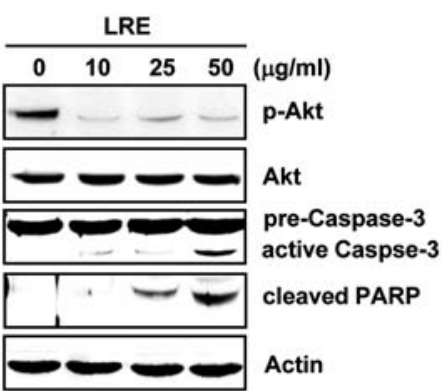

C

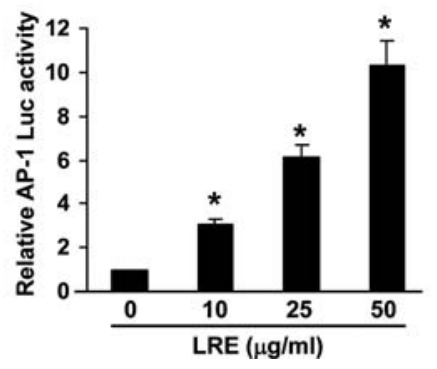

B

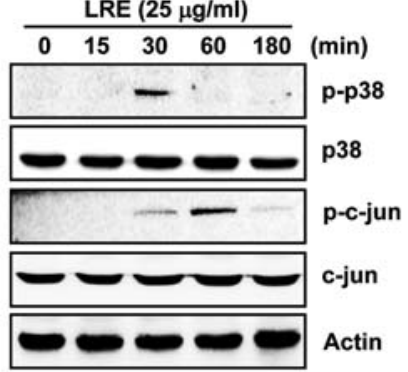

D

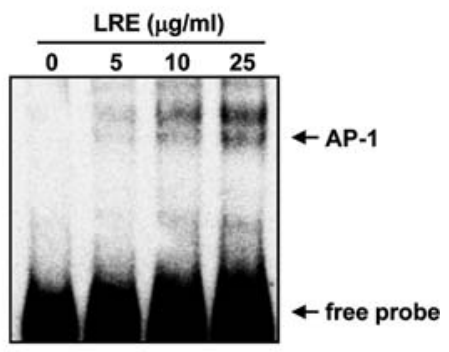

Figure 3. Increase in the level of active caspase-3, cleaved PARP, phosphorylation of p38 and c-Jun and AP-1 transcriptional activity after LRE treatment. (A) Cells were treated with specific concentrations of LRE for $24 \mathrm{~h}$ and lysates were subjected to Western blot analysis. Equal protein loadings were confirmed using anti-actin antibody. (B) Cells were treated with $25 \mu \mathrm{g} / \mathrm{ml}$ of LRE for the indicated time and cell lysates were subjected to Western blot analysis. (C) Cells were transiently transfected with a luciferase reporter construct using Lipofectamine 2000 reagent for $4 \mathrm{~h}$. After $18 \mathrm{~h}$ of additional incubation with LRE, cells were harvested in passive lysis buffer and the luciferase activities were measured by a luminometer using the luciferase assay system. ${ }^{~} \mathrm{P}<0.01$ vs. LRE-untreated B16F10 cells. (D) Nuclear extracts were prepared after treatment with indicated concentrations of LRE for $3 \mathrm{~h}$ and analyzed for DNA-binding activity of AP-1 using an AP-1 EMSA kit.

A

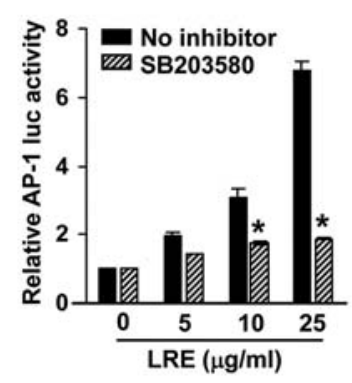

C

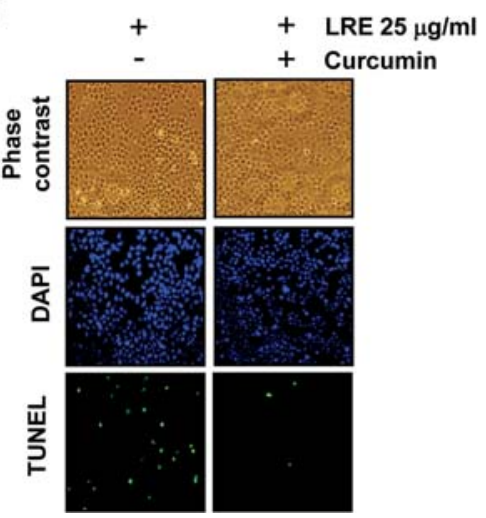

B

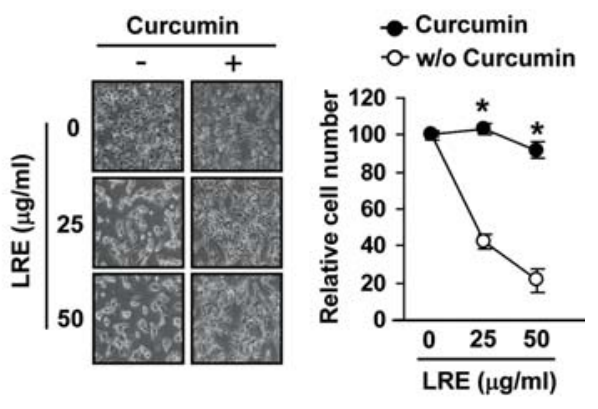

D
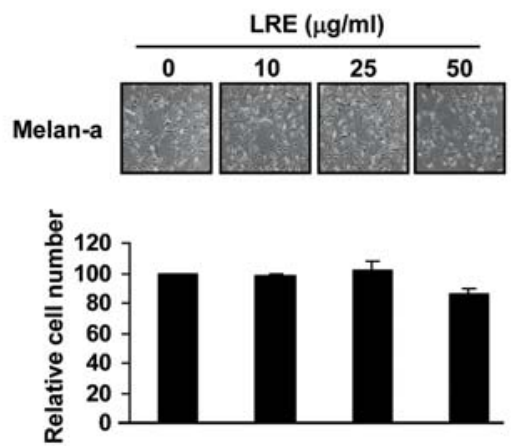

Figure 4. Effect of pretreatment with specific p38 inhibitor or AP-1 inhibitor on the LRE-mediated apoptosis. (A) Prior to the LRE treatment, cells were pretreated with p38 inhibitor (SB203580) at $2.5 \mu \mathrm{M}$ for $30 \mathrm{~min}$ and AP-1 transcriptional activities were measured using luciferase assay system. ${ }^{*} \mathrm{P}<0.01 \mathrm{vs.} \mathrm{SB203580-}$ untreated B16F10 cells. (B) Cells were pretreated with curcumin at $10 \mu \mathrm{M}$ for $2 \mathrm{~h}$ and then treated with $25,50 \mu \mathrm{g} / \mathrm{ml}$ of LRE for $24 \mathrm{~h}$. Morphological changes were observed under phase contrast microscope and relative cell number was determined by trypan blue dye exclusion. "P<0.01 vs. curcumin-untreated B16F10 cells. (C) Cells were harvested after LRE treatment with or without curcumin as described in (C). Green fluorescent apoptotic bodies were observed under fluorescence microscope. (D) Various concentrations of LRE were treated on the normal melanocyte cell line, Melan-a, for $24 \mathrm{~h}$. 
almost completely inhibited by pretreatment with $2.5 \mu \mathrm{M}$ of SB203580. This result indicates that AP-1 activation by LRE treatment was induced possibly through the p38 pathway. Next, we investigated whether suppression of the AP-1 activity by pretreatment with curcumin, as a well-known inhibitor of AP-1, blocks LRE-mediated apoptosis in B16F10 cells. As shown in Fig. 4B, cells pretreated with $10 \mu \mathrm{M}$ of curcumin prior to LRE treatment displayed high cell density and cell number compared to curcumin-untreated cells. Moreover, induction of apoptotic TUNEL-positive cells by LRE was considerably decreased by pretreatment with curcumin (Fig. 4C). This result indicates that AP-1 activation plays a crucial role in the LREmediated apoptosis in the B16F10 cells and AP-1 inhibition by curcumin maintains the cell survival in the presence of LRE. To determine the apoptotic effect of LRE in other cells, murine colon adenocarcinoma CT-26 cells were used. Similar to the results in B16F10 cells, LRE reduced cell viability of CT-26 cells in a dose- and time-dependent manner (data not shown). In the meantime, murine normal melanocyte Melan-a cells were less sensitive to the reduction of cell viability and induction of apoptosis by LRE treatment than B16F10 melanoma cells (Fig. 4D). Higher concentration of LRE and longer incubation times than those used for B16F10 cells were needed to elicit a similar decrease in cell viability and an increase in apoptosis (data not shown). These findings suggest that non-melanoma cells are more resistant to apoptosis caused by LRE treatment than cancer cells and therefore LRE may be used as anti-cancer agent.

The bulbs of Lycoris radiata have long been used as a folk medicine and some alkaloids isolated from this plant have been reported to exhibit immunostimulatory, anti-tumor, anti-viral and anti-malarial activities $(17,18)$. In this study, we demonstrated the effect of LRE treatment on the apoptosis induction in malignant melanoma cells and examine the possible underlying mechanisms. As demonstrated in the previous studies using lycorine as natural alkaoids isolated from Amaryllidaceae Lycoris, we observed that LRE can suppress melanoma cell growth and reduce cell survival via arresting cell cycle and inducing apoptosis. In particular, the present study is the first effort to specifically elucidate that phosphorylation of p38 and subsequent AP-1 activation contribute to the LRE-mediated apoptosis in melanoma cells.

Since tumor metastasis is a one of the fundamental properties of malignant cancer cells and a major cause of cancer mortality (19-21), our next focus on investigation is the effect of LRE on the modulation of metastatic potential of malignant cancer cells at the non-cytotoxic concentrations. In addition, we intend to demonstrate beneficial effect of this extract in a clinically relevant B16 mouse melanoma model and safety profile observed in vivo. These advanced studies are expected to provide strong support for the usage of LRE as a good candidate therapeutic agent against melanoma.

\section{Acknowledgements}

This study was supported by Technology Development Program for Agriculture and Forestry, Ministry of Food, Agriculture and Forestry, Republic of Korea, and also partly supported by Brain Korea 21 Program, the Ministry of Education and Human Resources, Korea.

\section{References}

1. Kim R, Tanabe K, Emi M, Uchida Y, Inoue H and Toge T: Inducing cancer cell death by targeting transcription factors. Anticancer Drugs 14: 3-11, 2003.

2. Darzynkiewicz Z: Apoptosis in antitumor strategies: modulation of cell cycle or differentiation. J Cell Biochem 58: 151-159, 1995.

3. Fisher DE: The p53 tumor suppressor: critical regulator of life and death in cancer. Apoptosis 6: 7-15, 2001

4. Kerr JF, Wyllie AH and Currie AR: Apoptosis: a basic biological phenomenon with wide-ranging implications in tissue kinetics. Br J Cancer 26: 239-257, 1972.

5. Raff MC: Social controls on cell survival and cell death. Nature 356: 397-400, 1992.

6. Savill J, Fadok V, Henson P and Haslett C: Phagocyte recognition of cells undergoing apoptosis. Immunol Today 14: 131-136, 1993.

7. Thompson CB: Apoptosis in the pathogenesis and treatment of disease. Science 267: 1456-1462, 1995.

8. Hayashi A, Saito T, Mukai Y, Kurita S and Hori TA: Genetic variations in Lycoris radiata var. radiata in Japan. Genes Genet Syst 80: 199-212, 2005.

9. Liu J, Hu WX, He LF, Ye M and Li Y: Effects of lycorine on HL-60 cells via arresting cell cycle and inducing apoptosis. FEBS Lett 578: 245-250, 2004

10. Li Y, Liu J, Tang LJ, Shi YW, Ren W and Hu WX: Apoptosis induced by lycorine in KM3 cells is associated with the G0/G1 cell cycle arrest. Oncol Rep 17: 377-384, 2007.

11. Liu XS, Jiang J, Jiao XY, Wu YE, Lin JH and Cai YM: Lycorine induces apoptosis and down-regulation of Mcl-1 in human leukemia cells. Cancer Lett 274: 16-24, 2009.

12. Wang L, Zhang XQ, Yin ZQ, Wang Y and Ye WC: Two new amaryllidaceae alkaloids from the bulbs of Lycoris radiata. Chem Pharm Bull 57: 610-611, 2009.

13. Angel P and Karin M: The role of Jun, Fos and the AP-1 complex in cell-proliferation and transformation. Biochim Biophys Acta 1072: 129-157, 1992.

14. Shaulian E and Karin M: AP-1 in cell proliferation and survival. Oncogene 20: 2390-2400, 2001.

15. Karin M: The regulation of AP-1 activity by mitogen-activated protein kinases. J Biol Chem 270: 16483-16486, 1995.

16. Whitmarsh AJ and Davis RJ: Transcription factor AP-1 regulation by mitogen-activated protein kinase signal transduction pathways. J Mol Med 74: 589-607, 1996.

17. Jin Z: Amaryllidaceae and Sceletium alkaloids. Nat Prod Rep 20: 606-614, 2003.

18. Jin Z: Amaryllidaceae and Sceletium alkaloids. Nat Prod Rep 24: 886-905, 2007

19. Jiang ZQ, Zhu FC, Qu JY, Zheng X and You CL: Relationship between expression of matrix metalloproteinase (MMP-9) and tumor angiogenesis, cancer cell proliferation, invasion, and metastasis in invasive carcinoma of cervix. Ai Zheng 22: 178-184, 2003.

20. Riedel F, Gotte K, Schwalb J, Bergler W and Hormann K: Expression of $92-\mathrm{kDa}$ type IV collagenase correlates with angiogenic markers and poor survival in head and neck squamous cell carcinoma. Int J Oncol 17: 1099-1105, 2000.

21. Talvensaari-Mattila A, Paakko P, Blanco-Sequeiros G and Turpeenniemi-Hujanen T: Matrix metalloproteinase-2 (MMP-2) is associated with the risk for a relapse in postmenopausal patients with node-positive breast carcinoma treated with antiestrogen adjuvant therapy. Breast Cancer Res Treat 65: 5561,2001 . 